\title{
An unusual giant spiral arc in the polar cap region during the northward phase of a Coronal Mass Ejection
}

\author{
L. Rosenqvist, A. Kullen, and S. Buchert \\ Swedish Institute of Space Physics, Uppsala, Sweden \\ Received: 1 August 2006 - Revised: 30 January 2007 - Accepted: 31 January 2007 - Published: 8 March 2007
}

\begin{abstract}
The shock arrival of an Interplanetary Coronal Mass Ejection (ICME) at 09:50 UT on 22 November 1997 resulted in the development of an intense $\left(\mathrm{D}_{s t}<-100 \mathrm{nT}\right)$ geomagnetic storm at Earth. In the early, quiet phase of the storm, in the sheath region of the ICME, an unusual large spiral structure (diameter of $\sim 1000 \mathrm{~km}$ ) was observed at very high latitudes by the Polar UVI instrument. The evolution of this structure started as a polewardly displaced auroral bulge which further developed into the spiral structure spreading across a large part of the polar cap. This study attempts to examine the cause of the chain of events that resulted in the giant auroral spiral. During this period the interplanetary magnetic field (IMF) was dominantly northward $\left(\mathrm{B}_{z}>25 \mathrm{nT}\right)$ with a strong duskward component $\left(\mathrm{B}_{y}>15 \mathrm{nT}\right)$ resulting in a highly twisted tail plasma sheet. Geotail was located at the equatorial dawnside magnetotail flank and observed accelerated plasma flows exceeding the solar wind bulk velocity by almost $60 \%$. These flows are observed on the magnetosheath side of the magnetopause and the acceleration mechanism is proposed to be typical for strongly northward IMF. Identified candidates to the cause of the spiral structure include a $B_{y}$ induced twisted magnetotail configuration, the development of magnetopause surface waves due to the enhanced pressure related to the accelerated magnetosheath flows aswell as the formation of additional magnetopause deformations due to external solar wind pressure changes. The uniqeness of the event indicate that most probably a combination of the above effects resulted in a very extreme tail topology. However, the data coverage is insufficient to fully investigate the physical mechanism behind the observations.
\end{abstract}

Keywords. Magnetospheric physics (Auroral phenomena; Magnetosheath; Magnetospheric configuration and dynamics)

Correspondence to: L. Rosenquist (lr@irfu.se)

\section{Introduction}

Auroral phenomena and their relation to the dynamics of the Earth's magnetosphere and the heliosphere have been studied over decades. In the current study, we report on some highly unusual, previously not documented, polar cap boundary deformations occurring during a Coronal Mass Ejection (CME). It appears as the sheath field of the 22 November 1997 CME passes by Earth. A huge auroral spiral reaching from the duskside oval far into the polar cap is formed near the end of the sheath period during strongly northward Interplanetary Magnetic Field (IMF) and very low solar wind pressure. Such an unusual poleward oval boundary change indicates large-scale deformations of the magnetotail that deviate strongly from previously studied cases. This CME event has been studied by several other authors, using it as a test case to examine a geomagnetic sudden commencement model (Lam and Rodger, 2001), focusing on airglow structures in the midlatitude ionosphere (Garcia et al., 2000), examining high-energetic ion beams near the velocity dispersion boundary of the high-latitude ionosphere (Popielawska et al., 2003), and being included in a smaller statistical study of stormtime substorms (Wu et al., 2004). Garcia et al. (2000) and Popielawska et al. (2003) observations show that the entire magnetosphere was in an extremely unusual state. Instead of putting the giant spiral as an outlier to the side we have asked ourselves which conditions of the solar wind and their history might have caused this unusual auroral form. Examining the magnetopause boundary, we try to get an understanding of how the magnetospheric toplogy might look like during this extraordinary deformation of the auroral oval.

The characteristics of CMEs and their impact on the Earth magnetosphere have been studied extensively. CMEs are caused by flares and erupting filaments at the surface of the sun and their velocities are often much higher than the surrounding solar wind thus forming an interplanetary shock structure. The sheath region between the shock and the main ejecta of the CME contains high magnetic fields.

Published by Copernicus GmbH on behalf of the European Geosciences Union. 
CMEs are highly geoefficient. It is mainly the $B_{z}$ southward part either in the sheath region or main ejecta that leads to a magnetic storm. Magnetic reconnection during southward IMF is the main process which leads to transfer of solar wind energy into the magnetosphere. Thus, ring current and auroral activity increase enormously after hours of strongly southward IMF.

The state of the magnetosphere during the northward phases of a CME has gained much less attention in the research community. The geomagnetic activity is much lower due to the low energy coupling between solar wind and magnetosphere for northward IMF. Although a CME northward phase does not lead to a magnetic storm, the magnetosphere does not automatically get into a quiet steady state. The existence of field-aligned (NBZ) currents in the dayside polar cap (Iijima et al., 1984), multiple convection cells (Weimer, 1995 ) and the frequent occurrence of auroral arcs poleward of the main oval (Valladares et al., 1994) show that, in general, the IMF northward phase is connected to strong activity in the polar cap.

The magnetosphere changes its characteristics in many aspects during periods of strongly northward IMF:

- The absence of tail reconnection leads to a decrease of the lobes (resulting in a contracted polar cap area).

- An increasing width of the low latitude boundary layer (LLBL) appears due to the tailward convection of newly closed field lines after high-latitude reconnection during northward IMF.

- The plasma sheet has a small dawn-duskward width but extends to high latitudes.

- Hasegawa et al. (2004) argue that during northward IMF large-scale surface waves are often observed along the magnetopause flanks that lead to plasma mixture in the tail via the Kelvin-Helmholtz instability. Fairfield et al. (2003) show that even solar wind pressure discontinuities may be responsible for magnetopause boundary motions. They propose that due to different propagation speeds of the pressure front in the magnetosheath and the boundary layer waves in the magnetosphere, a several Earth radii deep bulge develops at the magnetopause that propagates tailward with time.

- Fujita et al. (2005) report on a different effect caused by sudden solar wind pressure increases or decreases during northward IMF. With the help of MHD simulations they show that a pressure jump causes small pressure islands moving along the near-Earth plasma-sheet boundary from the flanks towards midnight during a transition phase. The small pressure islands are connected to transient field-aligned currents and a transient convection system that disappear when the new self-consistent state for new solar wind conditions are reached.
- During an extended period of northward IMF highspeed flows have been observed in the low-latitude magnetosheath that exceeds the velocity of the solar wind. It has been proposed by Chen et al. (1993) that the highspeed flows are caused by acceleration via magnetic field line tension in the draped field configuration during northward IMF and that they can lead to the excitation of surface waves.

- The probability for large-scale auroral arcs to occur poleward of the main oval is especially high after a prolonged time of strongly northward IMF, high IMF magnitude and solar wind speed (Kullen et al., 2002). As such solar wind conditions are typical for the northward IMF phases of a CME, luminous polar arcs appear frequently during CME events (Cumnock, 2005). The location and motion of polar auroral arcs are closely connected to large-scale deformations of the magnetotail that are caused by certain IMF conditions during northward IMF. While polar arcs separating from the dawn or dusk oval side are connected to large-scale plasma sheet twists (Kullen and Janhunen, 2004), arcs arising from the nightside oval are probably triggered by instabilities arising at the boundary of the plasma sheet (Rezhenov, 1995; Golovchanskaya et al., 2006).

In summary, although previous studies do show that in the CME northward phase the polar cap is active, and that large scale auroras, prominently polar arcs are frequently seen, a giant spiral developing from the nightside auroral oval has not yet been reported. Furthermore, an explanation to such a phenomenon is not readily available in literature. In this paper we examine in detail solar wind, auroral and magnetospheric data during this event. There are not enough measurements available to completely explain the spiral formation. However, combining our observations with results from previous studies during similar solar wind conditions we can discuss possible states of the magnetosphere that may lead to a giant auroral spiral. The remaining parts of the paper consist of a short instrument description (Sect. 2), presentation of observations by the Polar UV imager (Sect. 3.2) and Geotail (Sect. 3.3). In Sect. 4, the results are discussed with respect to possible magnetospheric deformations. A summary is given in Sect. 5 .

\section{Instruments}

The Polar UVI instrument is a two dimensional spatial imager in the far ultraviolet wavelength range on the Polar spacecraft which has been employed to monitor the Earth's auroral region (Torr et al., 1995). In addition to Polar UVI we will use Geotail measurements near the dawnside tail flank and solar wind data from the Wind spacecraft. The plasma measurements are made by the Solar Wind Experiment (SWE) (Ogilvie et al., 1995) and the magnetic field 

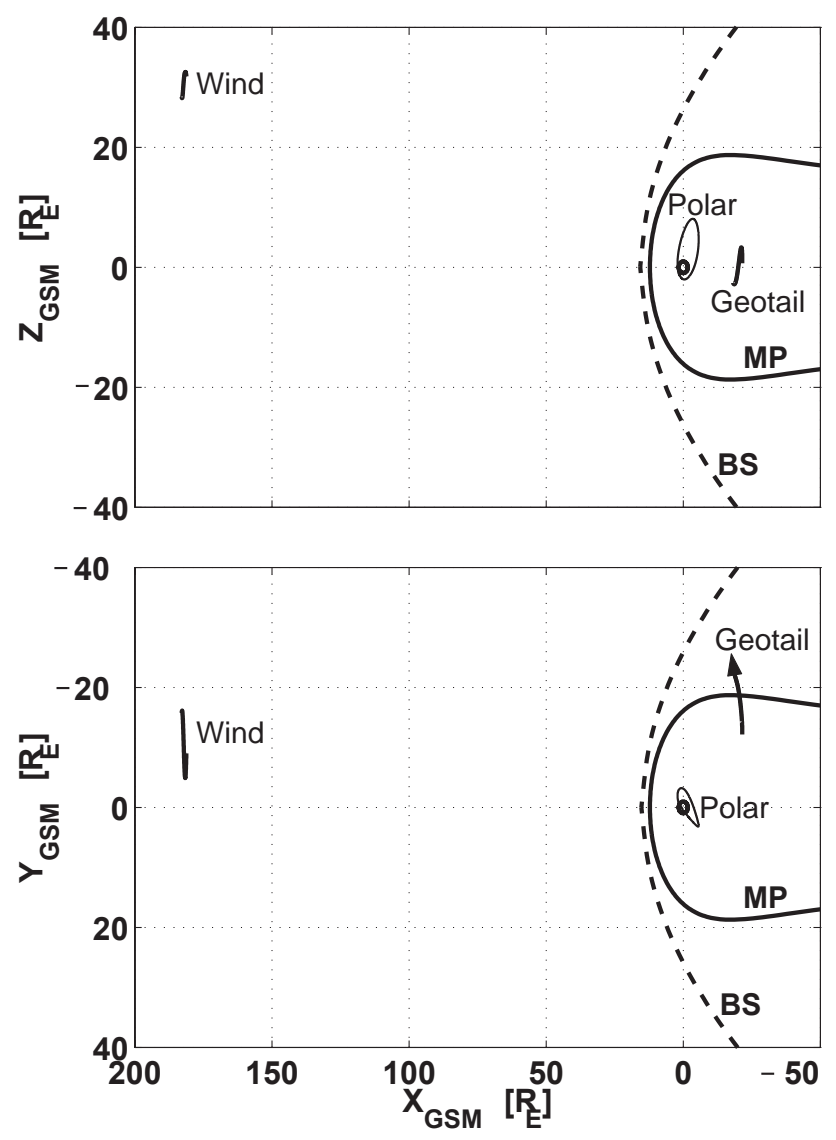

Fig. 1. Spacecraft configuration in the GSM (X-Z plane above, $X-$ Y plane below) coordinate system between 06:00 UT and 24:00 UT on 22 November 1997. The magnetopause location is predicted by the Shue et al. (1998) model using solar wind measurements from Wind at 19:00 UT.

measurements are 1-min resolution data obtained from the Magnetic Fields Investigation (MFI) (Lepping et al., 1995). The Geotail instruments used throughout this study are the Comprehensive Plasma Instrumentation (CPI) (Frank et al., 1994) for the plasma density data and the plasma velocity is calculated from low energy particle (LEP) measurements by the Solar Wind ion analyzer (LEP-SW) and ion energy analyzer (LEP-EA) (Mukai et al., 1994). The magnetic field values have been determined from the dual fluxgate magnetometers (Kokubun et al., 1994).

Figure 1 shows the trajectories of the Geotail and Polar spacecraft during the event considered in this study together with the position of the Wind spacecraft in the X-Y and X-Z GSM plane. The magnetopause (solid line) and bow shock (dashed line) position is predicted by the Shue et al. (1998) magnetopause model using solar wind measurements from WIND at 19:00 UT and the Bennett et al. (1997) bow shock model for average solar wind conditions.

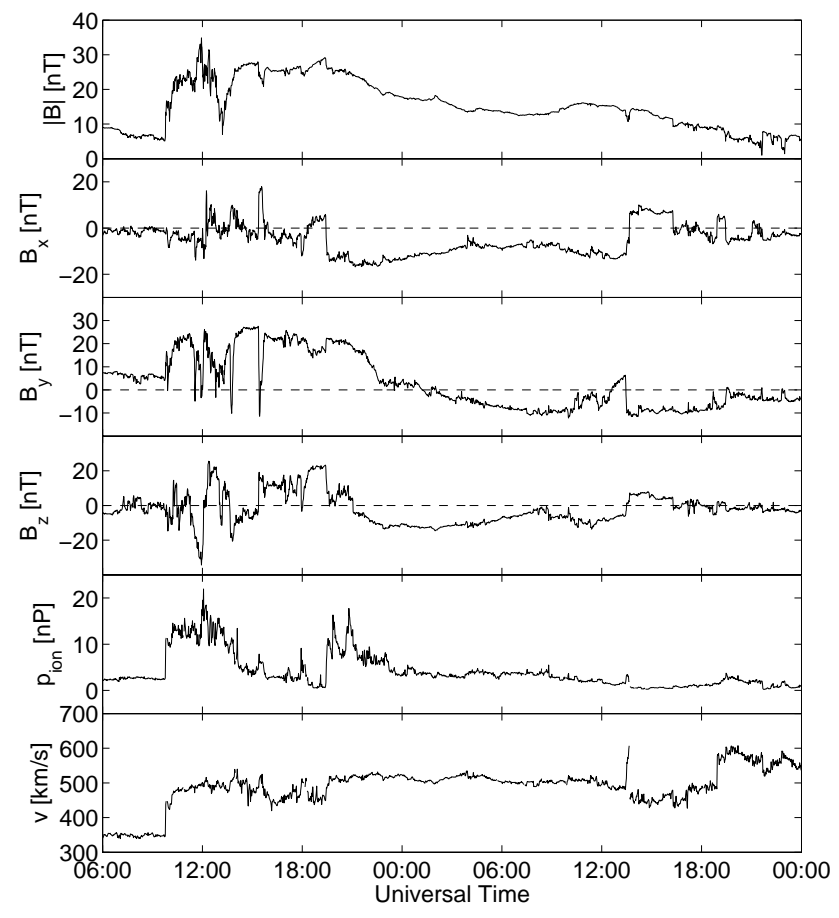

Fig. 2. Overview of Wind measurements on 22-23 November 1997. A propagation delay of $40 \mathrm{~min}$ have been added. From top to bottom, the figure shows the magnetic field magnitude, the magnetic field $B_{z}, B_{y}$, and $B_{x}$ GSM components (in nT); the solar wind pressure (in $\mathrm{nP}$ ); and the total ion bulk speed (in $\mathrm{km} / \mathrm{s}$ ).

\section{Observations}

\subsection{Solar wind observations}

An overview of solar wind conditions during the entire CME event is shown in Fig. 2. It shows WIND data near GSM $(\mathrm{x}, \mathrm{y}, \mathrm{z})=(181,-5,32) R_{E}$ from 06:00 UT on 22 November to 24:00 UT on 23 November 1997. The rows contain (from top to bottom) IMF magnitude, IMF $B_{x}, B_{y}, B_{z}$, solar wind pressure and speed. A propagation delay of $40 \mathrm{~min}$ has been added. The propagation time corresponds to an average time delay in the sheath region assuming a solar wind speed of $500 \mathrm{~km} / \mathrm{s}$ (average over the interval) and taking the radial distance from the spacecraft to Earth. As can be clearly seen in Fig. 3, the arrival of the Interplanetary CME foreshock is characterized by a sudden pressure increase. The sheath region between foreshock and main ejecta lasts for about $11 \mathrm{~h}$ until 19:30 UT (Mulligan et al., 1999). It is characterized by high solar wind flow $(500 \mathrm{~km} / \mathrm{s})$, high pressure and large magnetic magnitude. $B_{x}$ fluctuates around zero, $B_{y}$ has very high, positive values and $B_{z}$ is predominantly northward. Two excursions to strongly southward IMF appear at 11:23-12:12 UT and at 13:46-15:28 UT, respectively. The ion pressure is very high $(10-18 \mathrm{nPa})$ during the first $4.5 \mathrm{~h}$ and the last $1.5 \mathrm{~h}$ of the same sheath period, each time followed by a slow decrease to very low values. The decrease 


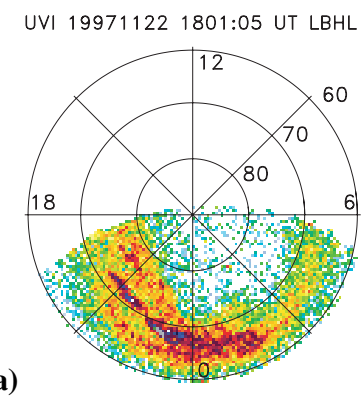

(a)

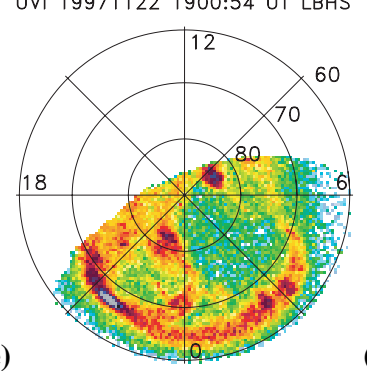

(e)

UVI 19971122 1914:41 UT LBHL

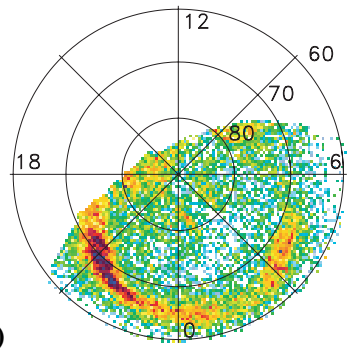

UVI 19971122 1820:43 UT LBHS

(b)

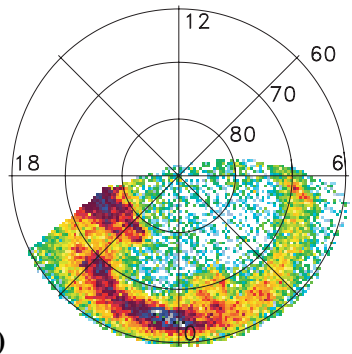

UVI 19971122 1903:39 UT LBHS

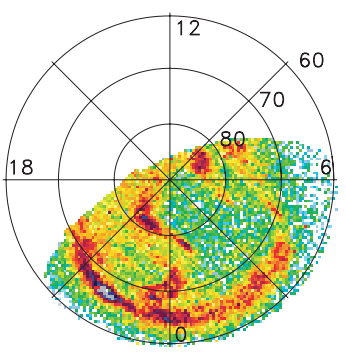

UVI 19971122 1928:30 UT LBHS

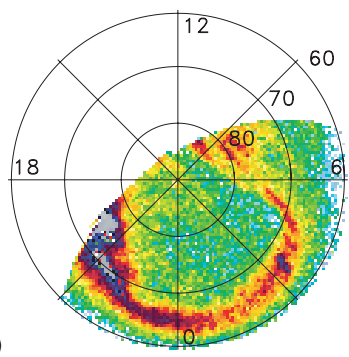

(c)

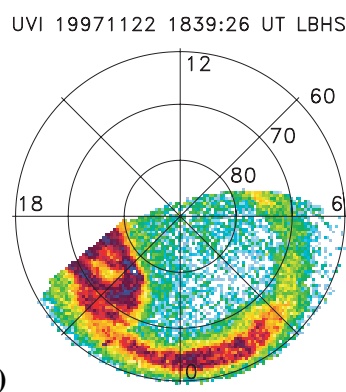

UVI 19971122 1907:02 UT LBHS

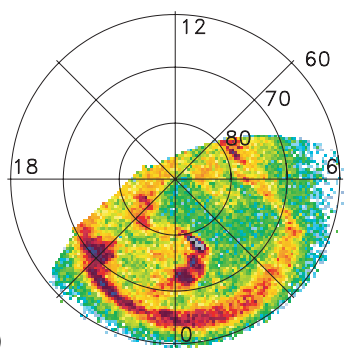

(h)

UVI 19971122 1940:46 UT LBHS

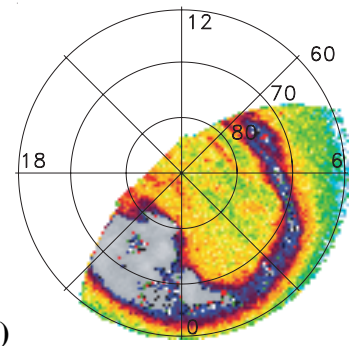

UVI 19971122 1851:42 UT LBHS

(d)

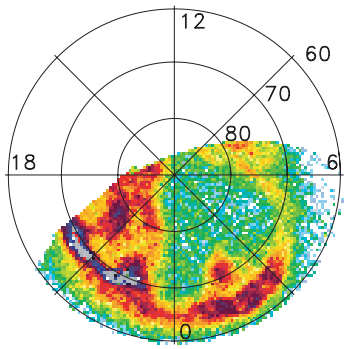

UVI 19971122 1910:06 UT LBHS

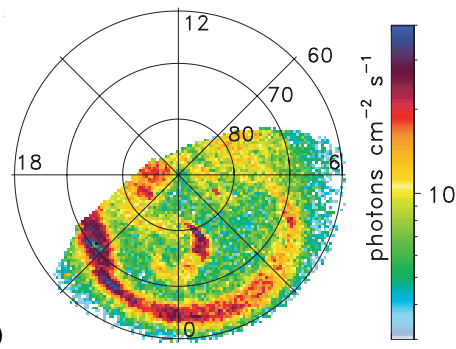

UVI 19971122 2100:11 UT LBHS

(l)

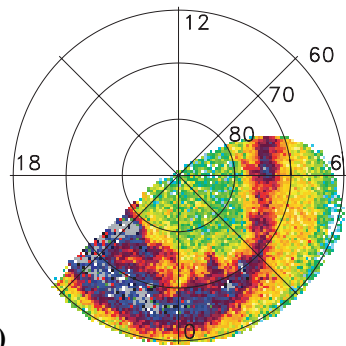

Fig. 3. Polar UVI images from 18:00 UT to 21:00 UT on 22 November 1997 in the CGM coordinate system.

during the sheath period is interrupted by small local maxima around 15:31 UT and 18:02 UT. Minimum values below $1.0 \mathrm{nPa}$ are reached $2.5 \mathrm{~h}$ before the sheath field has passed by the Earth (18:45-19:30 UT). The main ejecta arrives at 19:30 UT on 22 November at Earth and lasts for over 16h, characterized by a strong decrease of IMF $B_{z}$ to zero (after 21:10 UT to negative values) and a strong increase in dynamic pressure.

\subsection{Polar UVI observations}

The focus of this study lies on the strong changes of the polar cap boundary at the end of the CME sheath period leading to a giant auroral spiral in the middle of the polar cap. Fortunately, the auroral zone in the Northern Hemisphere is in the field of view of the Polar UVI imager over the entire sheath period such that the change in the auroral precipitation pattern can be followed in detail. Figure 3 shows Polar UVI images during the time span when the large-scale spiral event occurs.

As can be seen in Polar UVI (not shown here) the sudden commencement of the CME causes a first brightening at the dayside part of the aurora at 09:51 UT, within minutes of the onset time on the ground (Lam and Rodger, 2001). Several substorm activations occur during the sheath period. Neglecting all smaller intensifications, we find four clear substorm onsets: A first, strong substorm begins at 11:57 UT during the first longer IMF $B_{z}$ southward period. The next substorm starts during the second, less intense southward IMF period at 14:09 UT. Before this substorm has declined, a new substorm onset starts at 15:28 UT in connection with an IMF northturn. The onset of the last substorm during the sheath period takes place at 19:33 UT and is shown in Figs. 3j, k. It is probably triggered by the fast increase of the solar wind pressure associated with the main ejecta or the sudden turning of the IMF. The IMF drops from strongly northward to zero around the onset time. During the expansion phase of all four substorms, the poleward oval boundary moves to extreme high latitudes (around 80 degrees latitude) resulting in an extremely thick and active oval during recovery.

Several hundreds kilometer wide intrusions of auroral precipitation that move far into the polar cap occur at the recovery of the first substorm, at the recovery of the third substorm, 
and during the several hour long phase of relatively quietness before the last substorm of the sheath period starts. In all cases, the AE index has decreased to values below $300 \mathrm{nT}$ and IMF $B_{z}$ has been strongly northward for the last $40 \mathrm{~min}$ before the deformation occurs. The first two events can be classified as midnight arcs, as defined in Rezhenov (1995) and Kullen et al. (2002). In both cases polar arcs develop at the end of substorm recovery from a highly polewardly displaced bulge at the oval boundary and stretch within less than $15 \mathrm{~min}$ from the nightside oval towards the noon part.

The most remarkable deformation of the duskside polar cap boundary is seen during the quiet time phase after the second midnight arc has disappeared, (between 18:07 UT and 19:10 UT). As common during quiet times, the oval is rather small and thin as seen in Fig. 3a (62-68 degrees latitude at 00:00 MLT). During the hours with high solar wind energy and a constantly north-duskward IMF, the probability rises for an oval-aligned arc to develop at the duskside oval boundary. However, no classical oval-aligned arc with its tailward end attached to the nightside oval is seen. Instead, the duskside polar cap boundary moves in an irregular fashion strongly poleward (Figs. 3b-f), until it has passed the noon-midnight-line at 19:07 UT (Fig. 3g). During this deformation the inner parts of the oval become more and more empty from particles, and only the most equatorward and poleward parts of the oval stay visible, similar to a doubleoval structure, but rotated 90 degrees. Before the poleward oval boundary fades, it becomes detached from the nightside oval part. A giant spiral-like structure appears for some minutes that rolls up more and more (19:07-19:10 UT, Figs. 3gh), until it fades in the middle of the polar cap (Fig. 3i). Some small plasma fragments stay visible in the polar cap until 19:21 UT.

\subsection{Geotail observations}

Figure 4 shows an overview of Wind (red lines) and Geotail (black lines) plasma and IMF measurements in GSM coordinates from 06:00 UT to 24:00 UT on 22 November 1997. This interval is mainly characterized by the sheath region of the CME (09:47 UT-19:30 UT). The Geotail spacecraft moved outward through the dawn magnetosphere close to the equatorial plane (see Fig. 1). Using the T96 model with real solar wind input parameters (Tsyganenko, 1995) the Geotail position maps during the sheath period to the dayside part of the Northern Hemisphere. When the spiral appears, Geotail is topologically connected to the prenoon region of the auroral oval. However, the mapping results are not very realistic during this extreme event as will be discussed in the next chapter. Both Geotail and Wind data have been shifted in time to correspond to the the Polar UVI observations assuming a solar wind speed of $500 \mathrm{~km} / \mathrm{s}$ (average over the interval) and taking the radial distance from the spacecraft to Earth which resulted in a 40 min timeshift for Wind and a 6 min timeshift for Geotail.

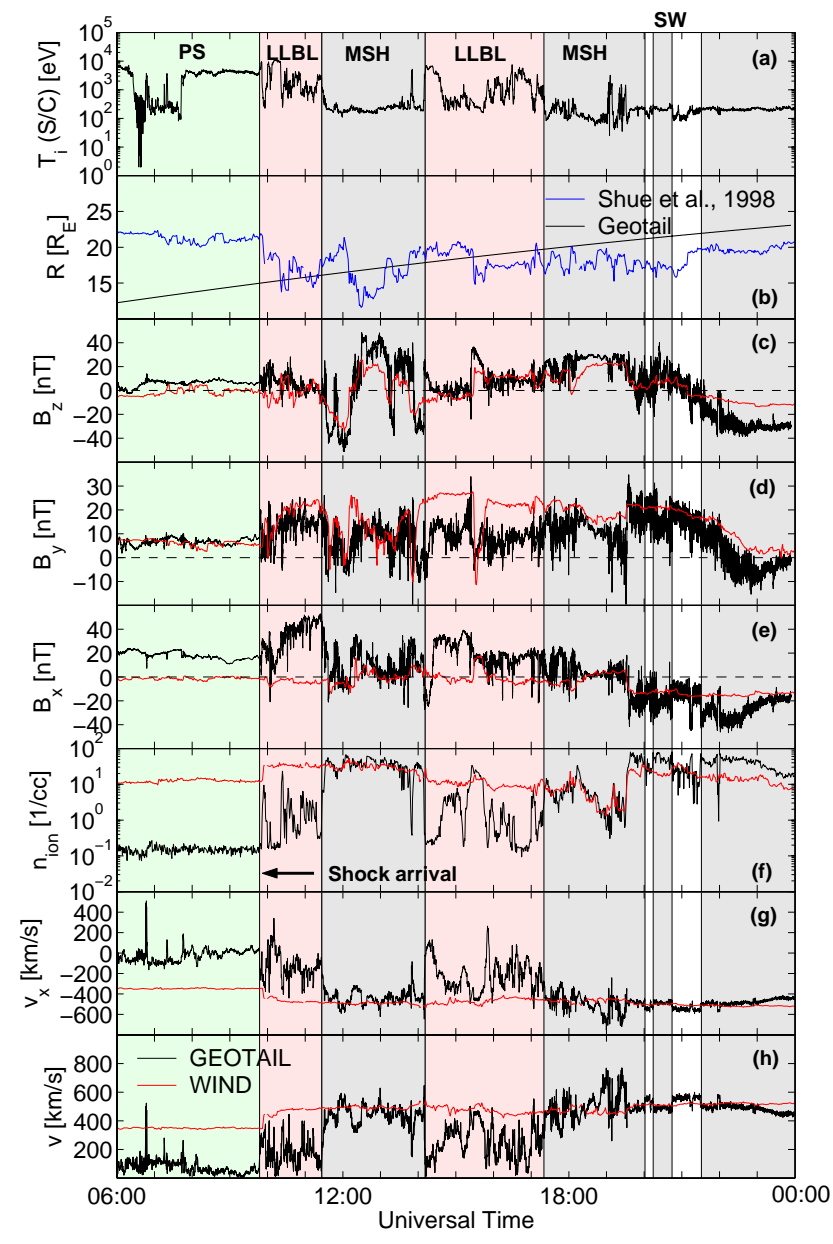

Fig. 4. Overview of Wind (red lines) and Geotail (black lines) measurements on 22 November 1997. The Wind data is propagated with $40 \mathrm{~min}$ and the Geotail observations are delayed by $6 \mathrm{~min}$ to correspond to the Polar UVI observations. From top to bottom, the figure shows the ion temperature in spacecraft coordinates $T_{i}$ (in $\mathrm{eV}$ ); the radial location of Geotail from the $\mathrm{x}$-axis and the radial dimension of the magnetopause (in $R_{E}$ ) predicted by Shue et al. (1998) model (blue line); the magnetic field $B_{z}, B_{y}$, and $B_{x}$ GSM components (in $\mathrm{nT}$ ); the ion density (in /cc); the GSM x-component of the velocity (in $\mathrm{km} / \mathrm{s}$ ); and the total ion bulk speed (in $\mathrm{km} / \mathrm{s}$ ). The different regions of the magnetosphere are shaded in different colours, light green for the Plasmasheet (PS), pink for the Low-Latitude Boundary Layer (LLBL), grey for the Magnetosheath (MSH), and white for the Solar Wind (SW). The arrival of the ICME shock is marked by an arrow.

The time period of interest is when Geotail observes accelerated plasma velocities far exceeding the solar wind flow at around 19:00 UT (see Fig. 4h). However, in order to determine the region of occurrence of these accelerated flows a longer time period is investigated before studying the details in higher time-resolution. The radius of the magnetopause (blue line in Fig. 4b) has been predicted from the Shue et al. (1998) model parameterized by the solar wind measurements 
made by Wind. This distance can be compared with the radial distance of Geotail from the GSM x-axis $\left(R^{2}=Y^{2}+Z^{2}\right)$ (black line in Fig. 4b) and can be used as a complement to the plasma measurmements to determine the geophysical region of Geotail.

At the beginning of the interval Geotail is in the inner magnetosphere observing high ion temperatures of $1-2 \mathrm{keV}$ (Fig. 4a), ion velocities around zero (Figs. $4 \mathrm{~g}$ and $\mathrm{h}$ ) and average ion densities of $0.2 / \mathrm{cc}$ (Fig. 4f), values typical for the low density, hot plasma sheet (shaded in light green in Fig. 4). The sudden increase in velocity and density observed by Wind associated with the arrival of the CME foreshock at 09:47 UT compresses the magnetosphere and Geotail observes a sharp change in flow velocity, ion density, and ion temperature. Geotail observes an enhanced tailward flow of $v_{x} \sim-200 \mathrm{~km} / \mathrm{s}$, the density increases to about $10-30 / \mathrm{cc}$ and the ions are somewhat colder. The x-component of the magnetic field at Geotail increases to about $50 \mathrm{nT}$ (Fig. 4e) which indicates a tailwardly stretched magnetic field. The prediction of the radial dimension of the magnetotail by Shue et al. (1998) suggests that Geotail is now close to the magnetopause. These observations suggest that Geotail has encountered the Low-Latitude Boundary Layer (LLBL, shaded in light pink in Fig. 4). The decreased temperature is due to magnetosheath cold ions $(<1 \mathrm{keV})$ entering the LLBL which is also responsible for the enhanced tailward flow. The plasma parameters are seen to be highly variable within this region. Whether these fluctuations are spatial variations within the region or due to an oscillatory motion of the magnetopause or the inner edge of the LLBL is not clear. However, the model predictions of the radius of the magnetopause suggest that it is rather an oscillatory motion of the magnetopause, in response to fluctuations in IMF $B_{z}$ and solar wind pressure, as $R$ (Fig. 4b) is seen to vary together with the plasma parameters measured at Geotail.

The IMF turns strongly southward at 11:23 UT (Fig. 4c) which would cause the magnetosphere to inflate due to the increase of open flux as a result of dayside reconnection which is supported by the prediction of the magnetopause model. However, Geotail measurements of plasma and magnetic field characteristics imply that the spacecraft has encountered the magnetosheath (MSH, shaded in grey in Fig. 4) rather than the inner magnetosphere. The erroneous prediction by the Shue et al. (1998) model can be explained by the occurrence of the first substorm onset. The substorm reduces the open flux in the tail due to tail reconnection and thus deflates the magnetosphere and counteracts the inflation due to an increase in open flux due to dayside reconnection (Milan et al., 2004). As the north-south component turns northward after about one hour the model prediction is now in agreement with Geotail being in the magnetosheath.

The solar wind density suddenly drops at 14:11 UT (Fig. 4f) which causes the magnetosphere to relax and the magnetopause to move outward across Geotail which encounters the LLBL again.
Geotail is mainly in the LLBL until about 17:20 UT. At this time Geotail is very close to the magnetopause according to the Shue et al. (1998) prediction. At 17:20 UT Geotail magnetic field and density observations suddenly align with Wind, this implies that the spacecraft is in the magnetosheath again. This is the region of the observed high speed flows as can be clearly seen in Fig. 4h. The ion bulk velocity at Geotail is at its most almost $60 \%$ higher than the measured solar wind flow by Wind. Geotail observes five periods of accelerated flows during this time period. However, we believe that this is a result of the region of high speed flows sweeping across Geotail rather than the accelerated flows being of bursty nature. One can note that the predicted dimension of the magnetotail oscillates during this period. The spacecraft seems to oscillate between the LLBL (seen as enhanced $B_{x}$, increased temperature and decreased density) and the MSH until the main ejecta of the CME hits at about 19:30 UT. The increase in solar wind density compresses the magnetosphere, thus Geotail abruptly encounters the outer regions of the magnetosheath were the density is now higher than the solar wind density (Phan et al., 1997). At 20:00 UT and 20:42 UT Geotail is actually crossed by the bow shock and encounters the solar wind for short time periods. The highly fluctuating density observations in these regions may be associated with waves in the foreshock region upstream of the bow shock (Phan et al., 1997).

Thus, this suggests that the accelerated flows are observed by Geotail in the magnetosheath although in a bounded region close to the magnetopause. This region continuously moves across Geotail which in the observations is seen as several periods of accelerated plasma velocity. This hypothesis is also strengthened by the magnetopause model predictions.

Let's now take a closer look at the characteristics of these flows and their connection to the auroral spiral described above. Figure 5 shows the ion temperature $(\mathrm{eV})$, the magnetic field $B_{z}$ component (nT), the solar wind ion pressure $(\mathrm{nPa})$ and the $\mathrm{AE}$ index (nT) giving the substorm activity, and the total bulk flow velocity $(\mathrm{km} / \mathrm{s})$ during the period between 17:00 UT and 21:00 UT. The red lines still correspond to Wind measurements and black to Geotail measurements. The data have been shifted in time to correspond to the measurements at Earth, as in Fig. 4. The vertical dashed lines marks the time of each image in Fig. 3 and are labelled with the corresponding syllable (a-1). As described in Sect. 3.3, the development of an irregularly growing bulge into the giant spiral (Figs. 3b-h) starts at 18:07 UT, only minutes after a clear peak in the solar wind pressure appeared (third panel in Fig. 5 at 18:02 UT) and the IMF has turned strongly northward (second panel). During the bulge development into a spiral (lines $b-h$ ), the solar wind pressure shows a bumpy decrease to minimum values below $1 \mathrm{nPa}$. A last small pressure jump is seen (line $\mathrm{h}$ ) while the spiral fades away. While the inner parts of the auroral bulge become void of particles, and its poleward boundary detaches from the 
nightside oval developing into a giant spiral between 19:00 and 19:10 UT (Fig. 3e-h), Geotail observes accelerated flows of about $750 \mathrm{~km} / \mathrm{s}$, exceeding the solar wind velocity with as much as $60 \%$. A sudden solar wind pressure increase around 19:26 UT to high values is connected to the onset of the next substorm, seen in Polar UVI around 19:28 UT (Fig. 3j-1), which is also indicated by a strong AE increase. The temperature (first panel in Fig. 5) increases drastically at 19:00 UT and 19:15 UT and is generally anti-correlated with the highspeed flows which suggests that Geotail observes a hotter region such as the LLBL during these periods.

Thus, the evolution of the spiral and the observation of high-speed flows occur simultaneously. In the following we will investigate a possible connection between these features.

\section{Discussion}

High-speed solar wind flows occur quite frequently, both in connection with CMEs and also over longer periods near solar minimum when the solar wind originating from coronal holes reaches the Earth. If this alone caused the giant spiral, perhaps by a Kelvin-Helmholtz instability developing at the flank of the magnetotail between the magnetosheath and the plasma sheet, then the spiral should be a common auroral form. As the appearance of the giant auroral spiral seems to be an extremely rare event, it is suggested that its formation is connected to a combination of different effects that are utterly caused by the solar wind. A large-scale change in the magnetotail topology due to strong IMF By during northward IMF or a deformation of the magnetopause caused by solar wind density fluctuations (maybe in connection with magnetopause surface waves) are possible candidates for causing the high-latitude auroral deformations. However, as there are not enough measurements available to get a global picture of the magnetospheric topology, the observations cannot be completely explained.

\subsection{IMF influence}

In Kullen et al. (2002) the importance of the IMF time history is demonstrated. The probability for auroral arcs to occur poleward of the main oval rises with the duration and strength of northward IMF before the arc actually develops. During northward IMF, the magnetosphere needs 20 to $40 \mathrm{~min}$ to adapt to new IMF conditions (Frank and Craven, 1988). This precondition is given in this event study. Each of the polewardly displaced auroral features appears after at least 40 minutes strongly north-duskward IMF.

The location of the midnight arcs and the spiral near the dusk oval side is expected from the given solar wind conditions. Observations (Tsyganenko, 1989) and MHD simulations (Walker et al., 1999; Kullen and Janhunen, 2004) show that during north-duskward IMF the plasma sheet is strongly twisted, leading to an expansion of the duskside

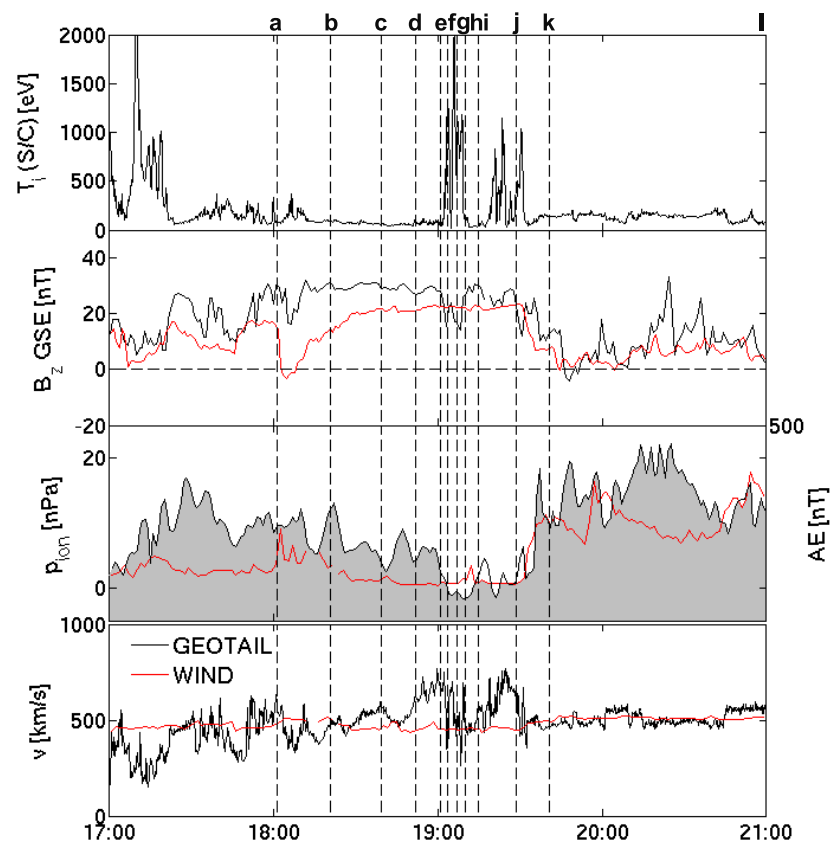

Fig. 5. From top to bottom, the ion temperature in spacecraft coordinates, the $B_{z}$ component of the magnetic field, the dynamic pressure and the $\mathrm{AE}$ index, and the ion bulk velocity. The red lines correspond to Wind measurements and the black to Geotail measurements. The data have been time shifted in the same manner as Fig. 4. The times of each of the images in Fig. 3 are marked by vertical dashed lines indicated by letters a-1.

plasma sheet to very high latitudes. Mapping the plasma sheet boundary to the northern ionosphere leads to a poleward displacement of the duskside closed field line boundary (Kullen and Blomberg, 1996). As discussed in Kullen and Janhunen (2004) the poleward boundary of the auroral oval does not necessarily coincide with the open-closed field line boundary. MHD simulations indicate that for constantly north-duskward IMF, the boundary of the main oval is found several degrees equatorward of the open-closed field line boundary. However, there is a high probability that auroral arcs occur poleward of the duskside oval (in the statistical study by Kullen et al. (2002) these arcs are of the oval-aligned and midnight arc type) which are suggested to belong to the expanded region of closed field lines (Makita et al., 1991; Kullen and Janhunen, 2004).

\subsubsection{Accelerated flows during northward IMF}

Geotail observed accelerated magnetosheath flows at the tailward flank of the magnetosphere at the same time as the spiral develops (Sect. 3.3). Such magnetosheath flows, exceeding the solar wind velocity have previously been observed by e.g. Howe and Binsack (1972), Petrinec et al. (1997), and Chen et al. (1993). Observations of accelerated flows near the magnetopause are often attributed to reconnection 


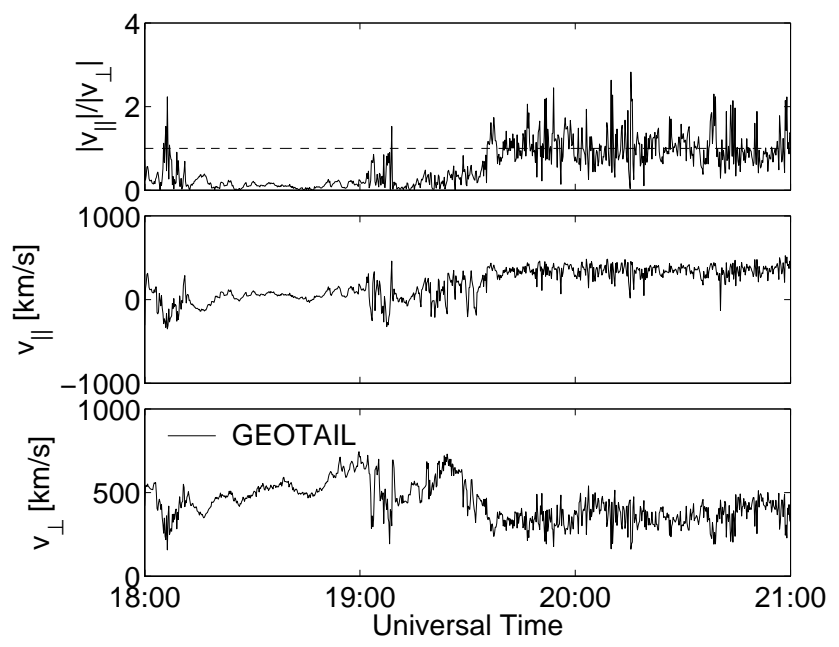

Fig. 6. From top to bottom, the ratio between the parallel and perpendicular velocity, the parallel velocity $(\mathrm{km} / \mathrm{s})$, and the perpendicular velocity $(\mathrm{km} / \mathrm{s})$.

processes and flux transfer events (Gosling et al., 1986). However, in our case the IMF is predominately northward and duskward which would imply high-latitude lobe reconnection on the Northern (Southern) Hemisphere on the duskward (dawnward) side. The observed accelerated flows in this study close to the equatorial region on the magnetosheath side of the magnetopause do not seem to be wellexplained by such a picture. The fast flows observed by Chen et al. (1993) appeared simultaneously at the dawn and dusk flanks during many hours of steady nothward IMF. Chen et al. (1993) proposed that such flows are accelerated in a sling-shot fashion by the magnetic field-line tension in the draped field configuration for northward IMF. Phenomenological, a solar wind magnetic flux tube will slow down across the bow shock near the subsolar point. It will continue to slow down as it approaches the magnetosheath and is then diverted around the flanks of the magnetopause as part of the magnetosheath flow. During the observation of these fast flows no strong substorm activity occurred and the absence of tail reconnection leads to the build up of magnetic tension as the portions of the flux tube outside the bow shock will continue to move with the solar wind plasma. Thus, if portions of the magnetic flux tube slow down near the subsolar magnetopause they must speed up somewhere else to catch up with the ends still remaining in the solar wind, thus at some point the flow will exceed the solar wind velocity. Chen et al. (1993) estimated quantitatively the importance of magnetic tension in accelerating magnetosheath plasma near the magnetopause. They estimated a maximum velocity of $750 \mathrm{~km} / \mathrm{s}$ gained from magnetic field acceleration which agrees well with the maximum velocity observed here. Thus, we suggest that the fast flows observed in our event are signatures of such a global field line draping that takes place at both flanks along the tail.
Another finding by Phan et al. (1997) is that the magnetosheath flow component tangential to the magnetopause is enhanced on approach to a low-shear magnetopause (corresponding to northward IMF) due to a strong increase of the flow component perpendicular to the magnetic field. Figure 6 shows the parallel and perpendicular flow components as well as the ratio between the two as measured by Geotail between 18:00 and 21:00 UT. It is evident that the accelerated flows are mainly perpendicular to the magnetic field which is an expected manifestation of the magnetic draping effect and consistent with the finding of Phan et al. (1997). The parallel velocity increases when the spacecraft is crossed by the magnetopause and exits the region of accelerated flow.

In addition, Chen et al. (1993) discuss the role of the magnetosheath flow in exciting surface waves on the magnetopause. However, in our case it is difficult to determine whether surface waves are exited or not due to the very dynamic solar wind. The magnetosphere frequently compresses and relaxes and Geotail encounters a number of different regions in a short time rather than continously observing one region. However, considering the similarites in the event observed by Chen et al. (1993) and in this study (strongly northward IMF, accelerated magnetosheath flow in the dawn side tail of similar amplitude) one might guess that such waves are present even in this case. What further strengthens such a guess is the observation of Pc-5 pulsations in the Greenland magnetometer chain (not shown). Magnetopause surface waves can be resonantly converted into shear Alfven waves inside the magnetosphere (Chen and Hasegawa, 1974) and this process is often considered to be the cause of Pc-5 pulsations at the magnetic field footpoints (Belmont and Chanteur, 1989). From this point, observations of the micropulsations can be used as direct diagnostics of the surface waves.

\subsection{Solar wind pressure influence}

Another common solar wind condition for the spiral events as well as the two midnight arcs occuring some hours before is a 5-10 nPa strong pressure decrease occurring for each event about 30-60 min before the aurora moves into the polar cap. The midnight arcs occur for a solar wind pressure level of 15 and $3 \mathrm{nPa}$, the spiral appears after the solar wind pressure has dropped from $10 \mathrm{nPa}$ to a minimum value of $0.5-1 \mathrm{nPa}$.

The multiple pressure changes during the 10-11 January 1997 event have been shown by Fairfield et al. (2003) to cause tailward propagating bulges of several $R_{E}$ depth along the magnetopause. As mentioned in the introduction, the 10-11 January 1997 event is similar to our case. It appears during the northward phase of a CME event with strongly decreasing density and simultaneous occurrence of multiple arcs. In that case, multiple arcs appear about 40 min after a last, rather small pressure change $(5 \mathrm{nPa})$ when the solar wind pressure has decreased considerably from its maximal level of over $50 \mathrm{nPa} 2 \mathrm{~h}$ before. The similarity between the event 
studied by Fairfield et al. (2003) and our event is the occurrence of unusual high-latitude auroral features during a prolonged northward IMF phase, and a sudden pressure change during a phase with extremely low solar wind pressure values. In our study, the most remarkable auroral feature, the giant auroral spiral, develops within an hour from a polewardly displaced bulge at the end of a several hour long decrease of the solar wind pressure to extreme low values around 0.5$1 \mathrm{nPa}$. As in the 10-11 January 1997 case, a clear pressure peak appears some time before the auroral spiral is seen. As the auroral pattern develops in an irregaluar fashion, it is difficult to determine the exact time delay between the pressure jump at 18:02 UT and the auroral spiral. A first bulge starts to develop only $5 \mathrm{~min}$ after the pressure peak, a clear poleward leap of the aurora is seen after about 30 minutes and the final spiral pattern is seen $60-65$ min after the pressure peak has passed.

If a solar wind pressure jump can cause a polewardly moving deformation at both magnetopause flanks, as suggested by Fairfield et al. (2003), this may change the entire magnetotail topology. During the time period, a duskside auroral bulge develops into the spiral (18:07-19:10 UT), a possible bulge-like deformation of the magnetotail will have propagated from $-23 R_{E}$ to $-280 R_{E}$ downtail (with a solar wind speed of $500 \mathrm{~km} / \mathrm{s}$ ). This means, the strongest deformation of the auroral precipitation pattern appears when a distortion of the magnetopause has reached extreme far tail regions. Assuming the multiple arcs in the 10-11 January 1997 event are caused by similar mechanisms as our spiral event, we may learn from looking at the changes in the magnetotail topology during polar auroral arc events. As shown in MHD simulations for polar auroral arcs (Kullen and Janhunen, 2004; Slinker et al., 2001) the closed field line region of the tail becomes extremely stretched during the formation of polar auroral arcs. The arc itself maps mainly to the far tail region. In the far tail region, a local bulge in the plasma sheet boundary could initiate an instability that may lead to a filamentation or bifurcation of the plasma sheet. The protrusion of plasma sheet filaments into the lobes have been observed during extended periods of northward IMF (Huang et al., 1989; Petrukovich et al., 2003) and have been connected to simultaneously observed multiple polar arcs (Huang et al., 1989).

\subsection{Formation of spiral}

The most remarkable deformation of the polar cap, the giant auroral spiral, occurs near the end of the sheath field period with very low solar wind pressure values and after nearly $4 \mathrm{~h}$ of constantly north-duskward IMF.

We know from previous observational studies that strong changes in the plasma population near the magnetotail flanks (Terasawa et al., 1997) and low-latitude boundary motions (Fairfield et al., 2003) or waves (Hasegawa et al., 2004) are expected after many hours of northward IMF. As discussed by Fairfield et al. (2003) surface waves might be initiated by the solar wind pressure changes, that are observed even in our case. Furthermore, the accelerated flows observed by Geotail could also lead to the generation of surface waves according to Chen et al. (1993) which is also supported by ground observations of Pc5 pulsations (see Sect. 4.1.1). However, we do not believe that this alone can explain the appearance of a large-scale spiral since no correlation between large-scale auroral features and surface waves has been identified so far (see e.g. Hasegawa et al., 2004).

The large-scale spiral does not resemble any polar arc type (theta aurora, oval-aligned arc, bending arc, midnight arc or multiple arc event). Polar auroral arcs need not necessarily be connected with the dayside oval (forming a true theta aurora), but all arc types are connected to the auroral oval with their nightside part (Kullen et al., 2002). Also, the spiral has a lifetime (very short) and an evolution (winding up in the middle of the polar cap before fading) that deviates strongly from all so far reported polar arc types. Furthermore, the spiral cannot be a substorm recovery feature. Although it develops from a double-oval like bulge, with the inner parts fading before the poleward part disappears, LANL particle data (not shown here) and the AE index show clearly that the event appears during hours of rather quiet conditions.

An auroral phenomenon of several hundred kilometres width is probably connected to large-scale topological changes in the magnetosphere. Mapping results with the T96 model by Popielawska et al. (2003) of velocity dispersed ion structures (appearing at the time period of the auroral spiral) show a rather unrealistic tail source region. As they mention, standard magnetosphere models do probably not cover such extreme solar wind conditions very well. Their results indicate a global deformation of the magnetosphere during the auroral spiral event. Thus, the mapping of the Geotail position to the prenoon auroral oval region may be unrealistic.

A several hour long strongly north-duskward IMF leads to a strong (seen from the Earth) anti-clockwise twist of the entire plasma sheet and a short tailward extension of the closed field line region (Kullen and Janhunen, 2004). The strongly polewardly displaced spiral will map to far tail regions that are partly detached from the main oval. Such a detachment of the far tail closed field-line region is possible, as shown in the case for a moving polar arc (Kullen and Janhunen, 2004).

We propose that the strong bulge-like deformation of the duskside auroral oval, developing into a giant auroral spiral in the polar cap, was caused by a tailwardly propagating bulge along the magnetopause, that was triggered by a pressure change, as suggested by Fairfield et al. (2003). Surface waves excited by accelerated magnetosheath flows, that are observed by Geotail at the time when the giant auroral spiral finally has developed, may indicate a positive interference of some kind between waves and boundary deformations that result in an extreme configuration of the tail. 


\section{Conclusions}

In this paper we have studied an extreme deformation of the polar cap boundary leading to a giant auroral spiral that exists for about $10 \mathrm{~min}$ in the middle of the polar cap. We examine this event with help of global auroral images by the Polar spacecraft and magnetotail flank observations by Geotail. Based on the available datasets, we suggest a possible scenario on how changes in the magnetospheric topology may be connected to the appearance of the giant spiral. We would like to point out, that the data coverage is insufficient to fully investigate the physical mechanism behind the observations. This paper is only a first step towards a better understanding on the dynamics of the magnetotail and its coupling to the auroral region during such an extreme deformation of the auroral oval.

The studied spiral event is unique. So far no other event with similar development has been reported. The spiral is neither similar to a small-scale auroral spiral due to its enormous width and size, nor a special form of polar arc as its nightside part is completely detached from the oval, nor a substorm (recovery) feature as it appears during quiet times with no sign of substorm activity at ionospheric or geosynchronous level.

We suggest that such a large-scale auroral deformation must be connected to large-scale topological changes in the magnetotail. From previous observations it is known that during north-duskward IMF the plasma sheet is strongly twisted which leads to a poleward displacement of the duskside closed field line boundary. In addition, the strong IMF northward component leads to a build up of magnetic flux in the absence of tail reconnection during this quiet period. Thus, enhanced magnetic draping results in accelerated flows along the magnetopause as observed by Geotail. Such flows enhance the local kinetic pressure and can excite surface waves on the magnetopause. In addition, the considerable reduction in external solar wind pressure could also cause a tailward propagating bulge of several $R_{E}$ depth along the magnetopause. Thus, such a combination could result in an extreme deformation of the far tail region leading to a filamentation or bifurcation of the plasma sheet. Such a protrusion of plasma sheet filaments into the lobes is connected to auroral forms poleward of the main oval in the ionosphere.

This paper demonstrates that the northward phase of CMEs may lead to spectacular auroral features in the polar cap that are probably connected to large-scale deformations of the magnetosphere. Studying such events is important as it helps to understand the solar wind-magnetosphere coupling during phases of high solar wind magnetic energy flux when no geomagnetic storms appear.

Acknowledgements. We are grateful to G. Parks for providing Polar UV data. We acknowledge CDAWeb for providing Wind and Geotail data to this study. We thank the PI's for the instruments, R. Lepping (Wind MFI) and K. Ogilvie (Wind SWE) of NASA/GSFC,
T. Mukai (Geotail LEP) of ISAS, L. Frank (Geotail CPI) of University of Iowa, and S. Kokubun (Geotail MGF) of STELAB Nagoya University.

Topical Editor I. A. Daglis thanks two referees for their help in evaluating this paper.

\section{References}

Belmont, G. and Chanteur, G.: Advances in Magnetopause KelvinHelmholtz Instability Studies, Physica Scripta, 40, 124-128, 1989.

Bennett, L., Kivelson, M. G., Khurana, K. K., Frank, L. A., and Paterson, W. R.: A model of the Earth's distant bow shock, J. Geophys. Res., 102, 26 927-26 942, doi:10.1029/97JA01906, 1997.

Chen, L. and Hasegawa, A. J.: A theory of pulsations 1: Steadystate exitation of field line resonance, J. Geophys. Res., 79, 1024-1032, 1974.

Chen, S.-H., Kivelson, M. G., Gosling, J. T., Walker, R. J., and Lazarus, A. J.: Anomalous aspectes of magnetosheath flow and of the shape and oscillations of the Magnetopause during an interval of strongly northward interplanetary magnetic field, J. Geophys. Res., 98, 5727-5742, 1993.

Cumnock, J. A.: High-latitude aurora during steady northward interplanetary magnetic field and changing IMF By, J. Geophys. Res., 110, A02 304, doi:10.1029/2004JA010867, 2005.

Fairfield, D. H., Farrugia, C. J., Mukai, T., Nagai, T., and Fedorov, A.: Motion of the duskflank boundary layer caused by solar wind pressure changes and the Kelvin-Helmholtz instability: 10-11 January 1997, J. Geophys. Res., 108, 1460, doi:10/1029/2003JA010134, 2003.

Frank, L. A. and Craven, J. D.: Imaging results from Dynamics Explorer 1, Rev. Geophys., 26, 249-283, 1988.

Frank, L. A., Ackerson, K. L., Paterson, W. R., Lee, J. A., English, M. R., and Pickett, G. L.: The Comprehensive Plasma Instrumentation (CPI) for the Geotail Spacecraft, J. Geomag. Geoelectr., 46, 23-37, 1994.

Fujita, S., Tanaka, T., and Motoba, T.: A numerical simulation of the geomagnetic sudden commencement: 3. A sudden commencement in the magnetosphere-ionosphere compound system, J. Geophys. Res., 110, A11 203, doi:10.1029.2005JA011055, 2005.

Garcia, F. J., Kelley, M. C., Makela, J. J., Sultan, P. J., Pi, X., and Musman, S.: Mesoscale structure of the midlatitude ionosphere during high geomagnetic activity: Airglow and GPS observations, J. Geophys. Res., 105, 18 417-18 428, 2000.

Golovchanskaya, I. V., Kullen, A., Maltsev, Y. P., and Biernat, H.: Ballooning instability at the plasma sheet-lobe interface and its implications for polar arc formation, J. Geophys. Res., 111, A11216, doi:10.1029/2005JA011092, 2006.

Gosling, J. T., Thomsen, M. F., Bame, S. J., and Russell, C. T.: Accelerated plasma flows at the near-tail magnetopause, J. Geophys. Res., 91, 3029-3041, 1986.

Hasegawa, H., Fujimoto, M., Phan, T.-D., Rème, H., Balogh, A., Dunlop, M. W., Hashimoto, C., and TanDokoro, R.: Transport of solar wind into Earth's magnetosphere through rolled-up KelvinHelmholtz vortices, Nature, 430, 755-758, 2004.

Howe, H. C. and Binsack, J. H.: Explorer 33 and 35 plasma observations of magnetosheath flow, J. Geophys. Res., 77, 3334-3344, 1972. 
Huang, C. Y., Craven, J. D., and Frank, A. L.: Simultaneous observations of a theta aurora and associated magnetotail plasmas, J. Geophys. Res., 94, $10137-10$ 143, 1989.

Iijima, T., Potemra, T., Zanetti, L., and Bythrow, P. F.: Large-scale Birkeland currents in the dayside polar region during strongly northward IMF: A new Birkeland current system, J. Geophys. Res., 89, 7441-7452, 1984.

Kokubun, S., Yamamoto, T., Acuna, M. H., Hayashi, K., Shiokawa, K., and Kawano, H.: The GEOTAIL magnetic field experiment, J. Geomag. Geoelectr., 46, 7-21, 1994.

Kullen, A. and Blomberg, L. G.: The influence of IMF By on the mapping between the Earth's magnetotail and its ionosphere, Geophys. Res. Lett., 23, 2561-2564, 1996.

Kullen, A. and Janhunen, P.: Relation of polar auroral arcs to magnetotail twisting and IMF rotation: A systematic MHD simulation study, Ann. Geophys., 22, 951-970, 2004, http://www.ann-geophys.net/22/951/2004/.

Kullen, A., Brittnacher, M., Cumnock, J. A., and Blomberg, L. G.: Solar Wind Dependence of the Occurrence and Motion of Polar Auroral Arcs: A Statistical Study, J. Geophys. Res., 107(A11), SMP 13-1, doi:10.1029/2002JA009245, 2002.

Lam, M. M. and Rodger, A. S.: A case study test of Araki's physical model of geomagnetic sudden commencement, J. Geophys. Res., 106, 13 135-13 144, 2001.

Lepping, R. P., Acuna, M. H., Burlaga, L. F., et al.: The Wind Magnetic Field Investigation, Space Sci. Rev., 71, 207-229, 1995.

Makita, K., Meng, C. I., and Akasofu, S. I.: Transpolar auroras, their particle precipitation, and IMF By component, J. Geophys. Res., 96, 14 085-14 095, 1991.

Milan, S. E., Cowley, S. W. H., Lester, M., Wright, D. M., Slavin, J. A., Fillingim, M., Carlson, C. W., and Singer, H. J.: Response of the magnetotail to changes in the open flux content of the magnetosphere, J. Geophys. Res., 109, A042 200, doi:10.1029/2003JA010350, 2004.

Mukai, T., Machida, S., Saito, Y., Hirahara, M., Terasawa, T., Kaya, N., Obara, T., Ejiri, M., and Nishida, A.: The low energy particle (LEP) experiment onboard the GEOTAIL satellite, J. Geomag. Geoelectr., 46, 669-692, 1994.

Mulligan, T., Russell, C. T., Anderson, B. J., Lohr, D. A., Rust, D., Toth, B. A., Zanetti, L. J., Acuna, M. H., Lepping, R. P., and Gosling, J. T.: Intercomparisons of NEAR and Wind interplanetary coronal mass ejections, J. Geophys. Res., 104, $28217-$ $28223,1999$.

Ogilvie, K. W., Chornay, D. J., and Frizenreiter, R. J.: SWE, a comprehensive plasma instrument for the WIND spacecraft, Space Sci. Rev., 71, 55-77, 1995.

Petrinec, S. M., Mukai, T., Nishida, A., Yamamoto, T., Nakamura, T. K., and Kokubun, S.: Geotail observations of magnetosheath flow properties, with simultaneous observations of the solar wind by the Wind spacecraft, Adv. Space Res., 20, 767-776, 1997.

Petrukovich, A. A., Baumjohann, W., Nakamura, R., Balogh, A., Mukai, T., Glassmeier, K.-H., Rème, H., and Klecker, B.: Plasma sheet structure during strongly northward IMF, J. Geophys. Res., 108, 1258, doi:10.1029/2002JA009738, 2003.
Phan, T. D., Larson, D., McFadden, J., Lin, R. P., Carlson, C., Moyer, M., Paularena, K. I., McCarthy, M., Parks, G. K., Réme, H., Sanderson, T. R., and Lepping, R. P.: Low-latitude dusk flank magnetosheath, magnetopause, and boundary layer for low magnetic shear: Wind observations, J. Geophys. Res., 102, $19883-$ $19895,1997$.

Popielawska, B., Sandahl, I., Joko, S., Stenuit, H., Romanov, S. A., and Zakharov, A. V.: Oxygen ion beams at the boundary of velocity dispersed ion structures in the high-latitude magnetosphere under northward interplanetary magnetic field with a large Bycomponent: INTERBALL-tail observations, Adv. Space Res., 31, 1363-1370, 2003.

Rezhenov, B. V.: A possible mechanism for theta aurora formation, Ann. Geophys., 13, 698-703, 1995, http://www.ann-geophys.net/13/698/1995/.

Shue, J. H., Song, P., Russel, C. T., Steinberg, J. T., Chao, J. K., Zastenker, G., Vaisberg, O. L., Kokubun, S., Singer, H. J., Detman, T. R., and Kawano, H.: Magnetopause location under extreme solar wind conditions, J. Geophys. Res., 103, 17 691-17 700, 1998.

Slinker, S. P., Fedder, J. A., McEwen, D. J., Zhang, Y., and Lyon, J. G.: Polar cap study during northward interplanetary magnetic field on 19 January 1998, Phys. Plasmas, 8, 1119-1126, 2001.

Terasawa, T., Fuijimoto, M., Mukai, T., et al.: Solar wind control of density and temperature in the near-Earth plasma sheet: Wind/Geotail collaboration, Geophys. Res. Lett., 24, 935-938, 1997.

Torr, M. R., Torr, D. G., Zukic, M., et al.: A far ultraviolet imager for the international solar-terrestrial physics mission, Space Sci. Rev., 71, 329-383, 1995.

Tsyganenko, N. A.: Global configuration of the magnetotail current sheet as derived from Geotail, Wind, IMP 8 and ISEE 1/2 data, J. Geophys. Res., 103, 6827-6842, 1989.

Tsyganenko, N. A.: Modeling the Earth's magnetospheric magnetic field confined within a realistic magnetopause, J. Geophys. Res., 100, 5599-5612, 1995.

Valladares, C. E., Carlson, H. C. Jr., and Fukui, K.: Interplanetary magnetic field dependency of stable Sun-aligned polar cap arcs, J. Geophys. Res., 99, 6247-6272, 1994.

Walker, R. J., Richard, R. L., Ogino, T., and Ashour-Abdalla, M.: The response of the magnetotail to changes in the IMF orientation: The magnetotail's long memory, Phys. Chem. Earth, 24, 221-227, 1999.

Weimer, D. R.: Models of high-latitude electric potential derived with a least error fit of spherical harmonic coefficients, J. Geophys. Res., 100, 19595-19608, 1995.

Wu, C. C., Liou, K., Lepping, R. P., and Meng, C. I.: Identification of substorms within storms, J. Atmos. Solar. Terr. Phys., 66, 125132, 2004. 